\title{
Young interferential experiments with filamentary light sources
}

Monica Mancenido, Gustavo Pozzi, Luciano Zunino, Mario Garavaglia

Monica E. Mancenido, Gustavo Pozzi, Luciano Zunino, Mario Garavaglia, "Young interferential experiments with filamentary light sources," Proc. SPIE 3190, Fifth International Topical Meeting on Education and Training in Optics, (8 December 1997); doi: 10.1117/12.294395

Event: Fifth International Topical Meeting on Education and Training in Optics, 1997, Delft, Netherlands 
Young interferential experiments with filamentary light sources

Mónica E. MANCEÑIDO, Gustavo POZZI, Luciano ZUNINO, and Mario GARAVAGLIA

\author{
Departamento de Física, Universidad Nacional de La Plata, and \\ Centro de Investigaciones Opticas (CIOp) \\ CC 124, Correo Central, 1900 La Plata, Argentina. \\ Fax: + 5421714341 . \\ E-mail: laserlab@pinos.com
}

\begin{abstract}
The van Cittert-Zernike theorem allows interpreting the coherence distribution of an electromagnetic field from simple interferential experiments, like Young experiments. The visibility of the interferential fringes is equal to the modulus of the complex degree of coherence of the optical field. In the experiments commented in this communication, an electrical bulb linear carbon filament and the focal line of a cylindrical lens illuminated by a collimated expanded He-Ne red laser light, were both assumed to be filamentary light sources in order to observe Young interferential fringes with each of them. The electric lamp is a polychromatic light source, while the laser is a monochromatic one. When a filamentary light source is parallel to the Young slits, the interferential fringes are well defined and highly contrasted. If the source is rotated in a plane parallel to that of the slits, the visibility of the interferential fringes monotonically decreases and reaches zero in case of perpendicularity. Interferential fringes were photographically recorded and the colour slides were electronically scanned and processed.
\end{abstract}

Keywords: Coherence, Filamentary Light Sources, Interference.

\title{
1. INTRODUCTION
}

The van Cittert-Zernike theorem states that "the complex degree of coherence, which describes the correlation of vibrations at a fixed point $P_{2}$ and a variable point $P_{1}$ in a plane illuminated by an extended quasi-monochromatic primary source, is equal to the normalized complex amplitude at the corresponding point $P_{1}$ in a certain diffraction pattern centered on $P_{2}$. This pattern would be obtained on replacing the source by a diffracting aperture of the same size and shape as the source, and on filling it with a spherical wave converging to $P_{2}$, the amplitude distribution over the wave-front in the aperture being proportional to the intensity distribution across the source.". This formulation of the theorem was borrowed from Born and Wolf ${ }^{1}$.

A classical experiment to exemplify the van Cittert-Zernike theorem is that made by Thompson and Wolf ${ }^{2}$ by using a simple optical instrument called the diffractometer. This apparatus was used to solve problems that arise from X-ray structure analysis. In the diffractometer the diffraction pattern produced by a small circular quasi-monochromatic incoherent light source is analysed with a system of two holes placed at $P_{1}$ and $P_{2}$. The distance between holes is varied to follow the amplitude distribution inside the diffraction pattern.

Recently, the same kind of analysis of a diffraction pattern was made by P. F. Meilán et. al. ${ }^{3}$ using two fixed parallel slits instead of a pair of moving holes. Both slits simultaneously cover the central disk and the first ring of the Airy pattern, and by selecting areas of the pattern with equal optical field amplitude, uniform visibility in the fringes is assured. An interesting result obtained was the observation of dislocation of Young fringes generated by the interference of light arriving to both slits from the central disk or from the first ring and those generated by the interference of light coming from the central disk and the first ring. The dislocation occurred because of the phase change in $\pi$ in the optical field degree of coherence due to a circular quasi-monochromatic incoherent light source.

As a formal extension of this experiment the Young interference pattern produced by filamentary light sources was observed, and will be described in this communication. Section 2 is devoted to the analysis of a polychromatic incandescent filamentary source while in section 3 the same experiment with a monochromatic coherent filamentary laser source is described. Changes in the Young interference pattern as a function of the slits rotation angle with respect to the filament are discussed. 


\section{POLYCHROMATIC INCANDESCENT FILAMENTARY LIGHT SOURCE}

The experimental set-up is shown in Figure 1. The linear filament of an electric bulb is along the $x_{f}$ axis, parallel to the plane $\left(x_{S}, y_{S}\right)$ that contains the pair of thin parallel slits and to the observation plane $\left(x_{o}, y_{o}\right)$. To describe the rotation of the slits around the $z$ axis, the coordinate systems $\left(x_{S}, y_{s}\right)$ and $\left(x_{O}, y_{O}\right)$, on the slits and observation planes respectively are introduced. The axis $x_{s}^{\prime}$, always parallel to the slits, forms an angle $\varphi$ with $x_{s}$. The incandescent filament acts as a polychromatic light source. To reduce its spectral width a red filter centred at $\lambda_{0}=635 \mathrm{~nm}$ with a full spectral width of $\Delta \lambda=130 \mathrm{~nm}$ was used. As the intensity of the fringes is low, observations were made through photographs taken with an $f=135 \mathrm{~mm}$ teleobjective focused at infinity, so the observation plane is that of the photographic film. Kodak E100 SW colour slide film was used. The interferential information was then electronically digitalized and processed.

The incandescent filament was assumed to be a bidimensional light source characterised by a function $S\left(x_{f}, y_{f}, t\right)$ that depends on the position across the source and on time. However, the source is very thin, that is to say, the ratio of its width to

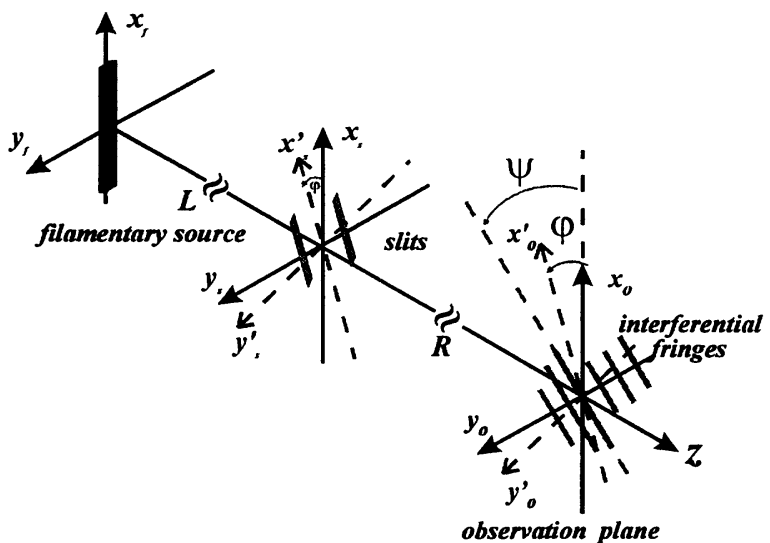

Fig. 1: Experimental set-up and definition of coordinate systems its length is of the order of $2 \times 10^{-3}$. The spectral content of the source can be introduced by Fourier transforming $S\left(x_{f}, y_{f}, t\right)$ in the customary fashion. As the light source is thin, it can be segmented along the $x_{\mathrm{f}}$-coordinate. The elements of length $\Delta x_{f}$ in which the source is divided are statistically equivalent. This means that all the elements are out of time correlation. In that case, the correlation function $\Gamma\left(x_{f}, x_{f}, \tau\right)$ can be expressed as:

$$
\Gamma\left(x_{f}, x_{f}, \tau\right)=\frac{1}{T} \int_{-\infty}^{\infty} d t S\left(x_{f}, t+{ }_{\tau}\right) S^{*}\left(x_{f}, t\right),
$$

which reaches its maximums at $\tau=0$ and vanishes when $\tau \rightarrow \infty$. Besides, as all the elements $\Delta x_{f}$ in the source are statistically equivalent: $\Gamma\left(x_{f}, x_{f}, \tau\right)=\Gamma(\tau)$, independent of $x_{f}$. Finally, $\Gamma(\tau)$ is related with the spectral light distribution of the source. Then, the light emitted by each segment of the source that traverses both slits, and after diffraction on them, impinges onto the photographic film, will interfere. The total irradiance due to the entire filament is equal to the superposition of the individual interferential patterns

$$
I\left(x_{o}, y_{o}\right)=\int d \omega\left(\frac{\omega}{2 \pi \tau}\right)^{2} \widetilde{S}_{0}(\omega) \int d x_{f}\left|K\left(x_{o}, y_{o}, x_{f}\right)\right|^{2} \text {, where: } \widetilde{S}_{0}(\omega)=\frac{1}{2 \pi} \int_{-\infty}^{\infty} d \tau \Gamma(\tau) e^{i \omega \tau},
$$

with $\omega=\frac{2 \pi}{\lambda} v, v$ the speed of light, and $K\left(x_{o}, y_{o}, x_{f}\right)=\int_{s l i s} d x_{s} d y_{s} \frac{\exp 2 \pi i(l+r) / \lambda}{l r}$.

$K\left(x_{O}, y_{O}, x_{f}\right)$ is the amplitude in a given point $\mathrm{P}\left(x_{O}, y_{O}\right)$ at the observation plane as a contribution from the segment of the filamentary source at $x_{f}$. If the dimensions of the source and the system of slits are much smaller than $L$ and $R$, as is actually the case, it results:

$$
l \cong L+\frac{\left(y_{s}-y_{f}\right)^{2}+\left(x_{s}-x_{f}\right)^{2}}{2 L} \quad \text { and: } \quad r \cong R+\frac{\left(y_{s}-y_{o}\right)^{2}+\left(x_{s}-x_{o}\right)^{2}}{2 R}
$$

These expressions are valid in the exponent of $K\left(x_{0}, y_{0}, x_{f}\right)$, while in its denominator they can reduced to their approximations $l=L$ and $r=R$.

Then, after the calculation of the integral, the square of the modulus of the function $K$ results:

$$
\left|K\left(x_{o}, y_{o}, x_{f}\right)\right|^{2}=\frac{4 b^{2} \lambda D}{L^{2} R^{2}} \operatorname{senc}^{2}(\pi q \alpha) \cos ^{2}(\pi \alpha)||_{-b / 2 \sqrt{\lambda D}}^{+b / 2 \sqrt{\lambda D}} d \xi \exp \left[\left.i \pi(\xi-\beta)^{2}\right|^{2},\right.
$$



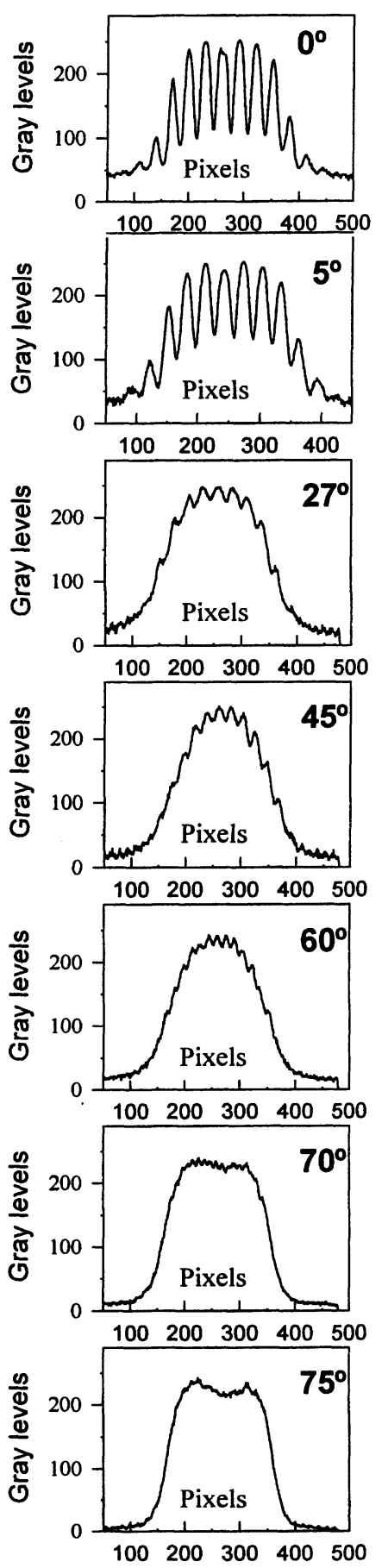

Fig. 2: Microdensitometric traces along the $x_{O^{-}}$axis for different rotations of the slits illuminated with a polychromatic filamentary light source. where $D=L R /(L+R), q=b / a, \alpha=\frac{a}{\lambda}\left(\frac{x_{o}}{R}-\frac{x_{f}}{L} \sin \varphi\right)$,

and $\beta=\sqrt{\frac{D}{\lambda}}\left(\frac{y_{o}}{R}+\frac{x_{f}}{L} \cos \varphi\right)$.

The integral in the expression of $\left|K\left(x_{O}, y_{O}, x f\right)\right|^{2}$, corresponds to the diffraction of light in the $x_{s}$ - direction. Formally, it is the diffraction in borders of the extremes of both slits, which resembles the diffraction by a rectangular aperture of width $\mathrm{d}$. However, as the primary purpose of this paper is to show the effect of the diffraction along the pair of slits, the integral can approximated by Heaviside functions. This approximation is equivalent to considering the Geometrical Optics description of the propagation of light in the $x_{s}{ }^{\prime}$ - direction. Then, the discussion that follows deals with the diffracted light at both thin slits neglecting the effects of the diffraction at their extremes. In other words, the model is restricted to the central part of the of the observation plane; from this position it is possible to observe the filament except its extremes. Then, the expression of $\left|K\left(x_{0}, y_{0}, x_{f}\right)\right|^{2}$ describes the distribution of the Young interferential fringes $\cos ^{2}(\pi \alpha)$ modulated by the diffracted light as $\operatorname{senc}^{2}(\pi q \alpha)$. The factor $q=b / a$ is insensible to the rotation of the pair of slits. Due to that, the amount of Young interferential fringes inside the zero order diffraction lobe is almost constant, in spite of the fact that the fringe spacing diminishes when the angle $\varphi$ increases. Finally, the spectral composition of the light affects the contrast across the interferential field. The spectral distribution of the red filtered filamentary incandescent source used in the experiment was not determined. However, a simple and qualitative interpretation of the experimental results can be proposed: As far as the spectral band width of the light is wide the contrast or visibility of the interferential fringes decreases as a function of their spatial position. So, the central Young fringes are always well defined while the marginal ones - those fringes located at the borders of the zero order diffraction lobe - are less and less contrasted when the angle $\varphi$ increases. Figure 2 contains a collection of microdensitometric traces obtained from digitalized interferograms recorded at various angles $\varphi$. It is clearly shown that the contrast of the Young fringes diminishes as a function of $\varphi$. When $\varphi$ is larger than $75^{\circ}$ the interferential effect of the parallel slits is obscured by the diffraction itself. In fact, observations at angles $\varphi$ close to $90^{\circ}$ resemble the diffraction pattern produced by a single slit of the same dimensions of each of the parallel pair.

\section{MONOCHROMATIC COHERENT FILAMENTARY LIGHT SOURCE}

As a natural extension of the experiment at Section 2, a monochromatic coherent filamentary light source was installed in the set-up at Figure 1. The focal line of a collimated laser beam passing through a cylindrical lens can be considered as a coherent monochromatic filamentary light source. The interference fringes produced when two thin parallel slits, situated in a plane perpendicular to the optical $z$ - axis, are rotated with respect to the source were studied.

The optical field produced by a long unidimensional coherent source is a cylindrical wave of amplitude proportional to $\exp (2 \pi r / \lambda) / r^{1 / 2}$, where $\lambda$ is the wavelength and $r$ is the distance to the source (measured perpendicularly to it). Let the filament be along the $x_{f}$-axis of a plane $\left(x_{f}, y_{f}\right)$, and the slits on $y_{s}{ }^{\prime}= \pm a / 2$ (parallel to the $x_{s}{ }^{\prime}$-axis) on a plane $\left(x_{S}, y_{s}\right)$, at a distance $L$ of the source, where the coordinate $x_{S}$ ' forms an angle $\varphi$ with $x_{s}$ (See Figure 1).

The phase difference on the slits plane is $\delta=(2 \pi s) / \lambda$, where $s=L\left[\left[1+\left(y_{S} / L\right)^{2}\right]^{1 / 2}-1\right]$ , and $r=L+s$ (See Figure 3). If the slits are small with respect to $L$, then $y_{s}<<L$ on the slits, 


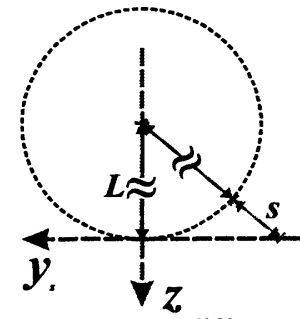

Fig. 3: Phase difference and using the binomial expansion: $\delta=\left(2 \pi y_{s}^{2}\right) / \lambda L$, and $r^{1 / 2}$ can be considered constant in the denominator.

The field amplitude on the slits plane is then proportional to:

$$
A\left(x_{s}^{\prime}, y_{s}^{\prime}\right)=\frac{\exp \left(i \pi\left(x_{s}^{\prime} \sin \varphi+y^{\prime} \cos \varphi\right)^{2}{ }_{s}\right.}{\lambda L}
$$

At a distance $R$ of the slits, on the observation plane with coordinates $x_{O}{ }^{\prime}-y_{O}$ ' parallel to $x_{s}-y_{s}$ ', or $x_{o^{-}} y_{o}$ parallel to $x_{f} y_{f}$, (figure \#), the optical field can be calculated using the HuygensKirchhoff formula:

$$
A\left(x_{o}^{\prime}, y_{o}^{\prime}\right)=\int_{\text {slits }} \frac{\exp \left(i \delta\left(x_{s}^{\prime}, y_{s}^{\prime}\right)\right) \exp (i 2 \pi \rho / \lambda)}{\rho}
$$

where $\rho=R\left[1+\left(x_{O}{ }^{\prime}-x_{S}{ }^{\prime}\right)^{2} / R^{2}+\left(y_{O}{ }^{\prime}-y_{S}{ }^{\prime}\right)^{2} / R^{2}\right]^{1 / 2}$

If the silts are small with respect to $R$, then $\left(x_{O}{ }^{\prime}-x_{S}{ }^{\prime}\right) / R$ and $\left(y_{O}{ }^{\prime}-y_{S}\right) / R$ are $\ll<1$, so using the binomial expansion $\rho=R\left[1+1 / 2\left(x_{O}{ }^{\prime}-x_{S}{ }^{\prime}\right)^{2} / R^{2}+1 / 2\left(y_{O}{ }^{\prime}-y_{S}{ }^{\prime}\right)^{2} / R^{2}\right]$ which in the denominator can be considered constant.

$$
A\left(x_{o}^{\prime}, y_{o}^{\prime}\right)=\int_{\text {lits }} d x_{s}^{\prime} d y_{s}^{\prime} \exp i \pi\left[\frac{\left(x_{s}^{\prime} \sin \varphi+y_{s}^{\prime} \cos \varphi\right)^{2}}{\lambda L}+\frac{\left(x_{o}^{\prime}-x_{s}^{\prime}\right)^{2}+\left(y_{o}^{\prime}-y_{s}^{\prime}\right)^{2}}{\lambda R}\right]
$$

Rewriting the term in square brackets changing variables, and considering the slits lineal (width null, length infinite), the integral can be solved to yield:

$$
A\left(x_{o}^{\prime}, y_{o}^{\prime}\right)=\frac{1}{\sqrt{L / R+\sin ^{2} \varphi}} \cos \frac{\pi a}{\lambda R}\left(\frac{\sin \varphi \cos \varphi}{L / R+\sin ^{2} \varphi} x_{o}^{\prime}-y_{o}^{\prime}\right)
$$

The sinusoidal variation of the field in the coordinates of the observation plane means there are fringes of equation:

$$
y_{o}^{\prime}=\frac{\sin \varphi \cos \varphi}{L / R+\sin ^{2} \varphi} x_{o}^{\prime}+n \frac{2 \lambda R}{a}
$$

or, in terms of $x_{o}$ and $y_{o}$ :

$$
y_{o}=(1+R / L) \operatorname{tg} \varphi x_{o}+n \frac{2 \lambda R}{a} \frac{1+R / L \sin ^{2} \varphi}{\cos \varphi}
$$

The slope of this equation is $\operatorname{tg} \psi=(1+R / L) \operatorname{tg} \varphi$, where $\psi$ is the angle formed by the fringes with respect to the source.

The experimental set-up sketched in Figure 1 was used. The beam of a red He-Ne laser $(\lambda=633 \mathrm{~nm})$ was expanded and collimated to about $5 \mathrm{~cm}$ in diameter. The plane face of a cylindrical lens was aligned perpendicularly to the beam. The same slits used in the previous part were aligned parallel to the focal line, $25 \mathrm{~cm}$ away from it. Photographs were taken with the film of a lensless camera at different distances $R$ to the slits, at each of which the slits were rotated from $0^{\circ}$ to $90^{\circ}$. Figure 4 contains a collection of 40 examples of these interferograms. They were photographed at five different values of the ratio $R / L$ and at eight angles $\varphi$. The angle $\psi$ was measured in them, and is plotted against $\varphi$ in Figure 5. The deduced relation between $\psi$ and $\varphi$ is also plotted for the corresponding values of $R / L$. When the slits are either parallel or perpendicular to the source, the fringes are parallel to the slits, at all distances. However, for other angles, at shorter distances the fringes tend to follow the slits, while at longer distances the fringes become almost perpendicular to the source for smaller rotations of the slits. 


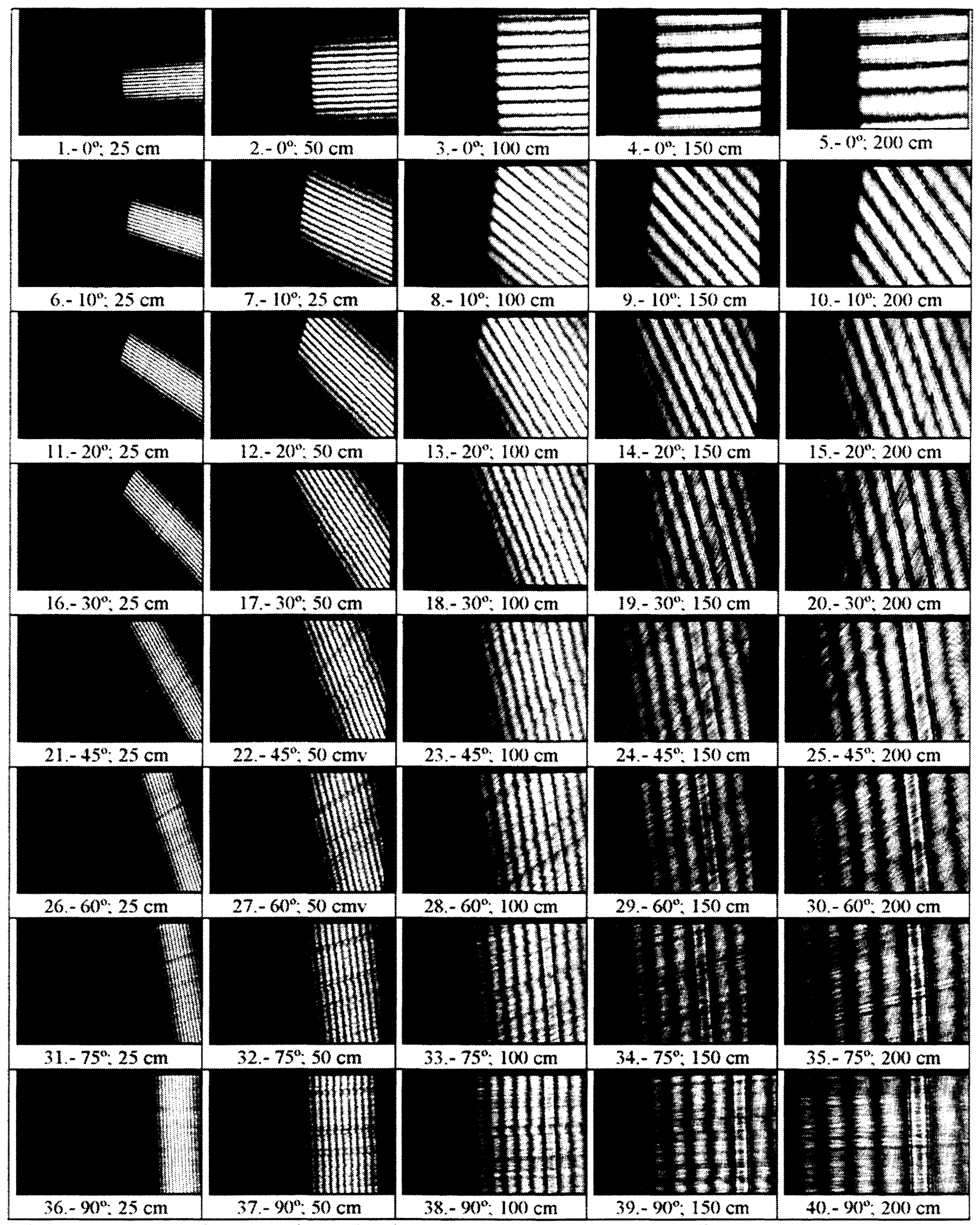

Fig. 4: Interferograms for a monochromatic coherent filamentary source, taken at different observation distances with different rotations of the slits. 


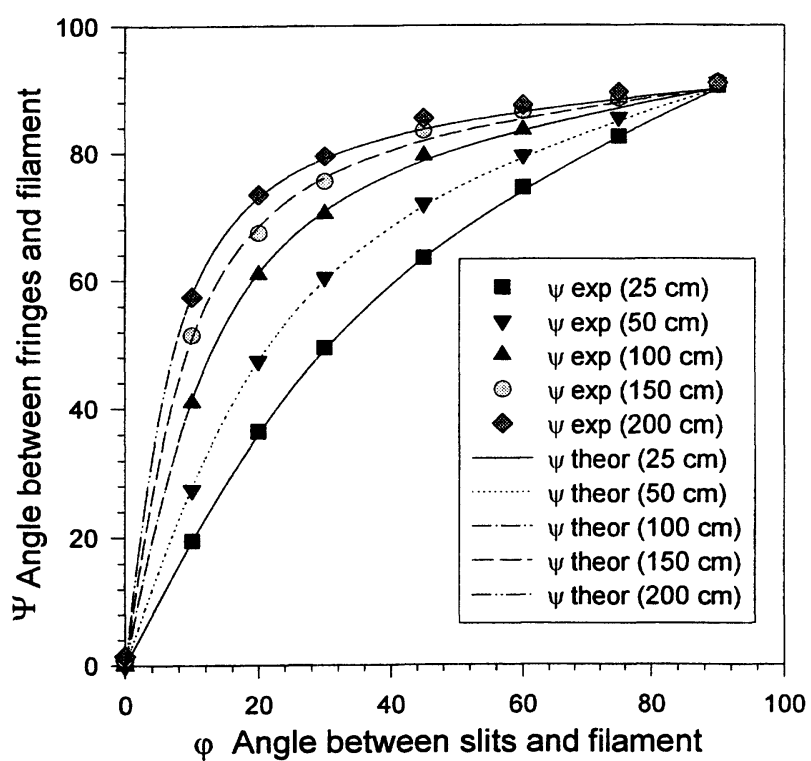

Figure 5: Angle $\psi$ between the fringes and the filament vs. angle $\varphi$ between the slits and the filament for different observation distances, and the calculated relation between them.

\section{CONCLUSIONS AND REMARKS}

The interferential experiments to observe incoherent polychromatic and coherent monochromatic filamentary light sources described in the previous sections were analysed using simple models. These models can be expanded in order to discuss much more detailed descriptions of the experimental facts observed. However, as the experiments described in this communication are simple to install and to interpret, the authors consider that it is possible to demonstrate them in theoretical and practical courses on Optics. Particularly, students can use pairs of parallel slits photographically prepared in slide format to observe the same effects reported in this communication. The incandescent light can be observed with the naked eye, placing the slits in front of it, while the laser source is intense enough to observe using a piece of white paper as screen at different distances.

Two comments follow related with the traces at Figure 2 and the pictures at Figure 4. The central fringe in the trace corresponding to the interferogram taken at $\varphi=0^{\circ}$, shows a clear reduction in intensity. A simple explanation is intended. As the pair of parallel slits are $37,5 \mu \mathrm{m}$ wide and $237,5 \mu \mathrm{m}$ spaced, they are sandwiched by two protective thin sheets of glass. The same effect was observed in diffraction using a similarly sandwiched single slit. Then, such a reduction in intensity could be produced by Fizeau interference in the sheets of glass. The second comment is related with pictures at Figure 4. They clearly display other, narrower interference fringes superimposed on the Young fringes. Calculations make it possible to assure that they correspond to linear Newton "rings" produced by internal reflections at the cylindrical lens.

\section{ACKNOWLEDGEMENTS}

The authors are very grateful to Dra. Lía M. Zerbino and to D. Graciela Benitez for their help in image processing. The authors are thankful for the partial financial support from Consejo Nacional de Investigacione Científicas y Técnicas and Universidad Nacional de La Plata, Argentina. CIOp depends on Consejo Nacional de Investigaciones Científicas y Técnicas and Comisión de Investigaciones Científicas de la Provincia de Buenos Aires, Argentina.

\section{REFERENCES}

1. M. Born and E. Wolf, Principles of Optics, Pergamon Press, Oxford, ( $5^{\text {th }}$ edition), p. 510, 1975

2. B. J. Thompson and E. Wolf, "Two-beam interference with partially coherent light" Journal of the Optical Society of America, 47, 895-902 (1957)

3. P. F. Meilán, G. Torchia, L. Errico, L. M. Zerbino, and M. Garavaglia, "Análisis de la distribución de coherencia mediante una experiencia interferencial de Young" (In Spanish), Actas of the VI Simposio Nacional de Física Experimental y Aplicada, y V Encuentro Nacional de Educación en Física Experimental, Temuco - Chile, pp. 433-438 (1996) 